\title{
Implementation of Outpatient Pharmacist-led Hypertension Management for Under- Resourced Patients: A Pilot Study
}

Brittany Stewart, RD, PharmD ${ }^{1}$; Aaron Brody, MD'; Candice L. Garwood, PharmD, FCCP3; Liying Zhang, PhD ; Phillip D. Levy, MD, MPH

${ }^{1}$ Department of Pharmacy Practice, Eugene Applebaum College of Pharmacy and Health Sciences, Wayne State University ${ }^{2}$ Department of Emergency Medicine, School of Medicine and Integrative Biosciences Center, Wayne State University ${ }^{3}$ Department of Pharmacy Practice, Eugene Applebaum College of Pharmacy and Health Sciences, Wayne State University, Department of Pharmacy, Harper University Hospital, Detroit Medical Center

${ }^{4}$ Epidemiology Research Design Core, Department of Family Medicine and Public Health Sciences, Wayne State University

${ }^{5}$ Department of Emergency Medicine, School of Medicine and Integrative Biosciences Center, Wayne State University

\begin{abstract}
Background: The purpose of this study was to implement and evaluate a pharmacist-led hypertension (HTN) program for underresourced patients discharged from the emergency department (ED) or screened at community health events who are lacking a regular primary care provider $(P C P)$ relationship.

Methods: This was a single arm, prospective, pilot study to recruit patients from the Detroit Medical Center (DMC) Sinai Grace Hospital (SGH) ED and community health events. The outpatient pharmacist-led transitional care clinic (TCC) was implemented through a collaborative practice agreement (CPA) with ED physicians. Eligible patients 18 to 60 years with elevated blood pressure (BP) $(>140 / 90$ $\mathrm{mmHg}$ ) and lacking a PCP relationship were referred to the TCC for HTN management. The primary outcome measure was change in systolic and diastolic BP (SBP and DBP). Difference in BP values was evaluated using Wilcoxon signed Ranks test and descriptive statistics were used to explain demographic data.

Results: There were 116 patients enrolled May 2017 to August 2018; 44 (37.9\%) completed visit one [cohort 1], 30 (25.9\%) completed at least three visits [cohort 2], and 16 (13.8\%) completed five visits [cohort 3]. Most patients were African American (AA) 97.7\%, 47.8\% were male, and an average of 42.11 (SD 9.70) years. For cohorts 2 and 3, there was significant reduction in BP between TCC visits one and two and the reduction was maintained through five visits for patients that remained in the study. Patients who completed five visits $(n=16)$ showed a significant change from visit one to visit five in SBP of $-23 \mathrm{mmHg}(p=0.002)$ and achieved BP goal with an average SBP $139 \mathrm{mmHg}$ (SD 19.33) and DBP $90 \mathrm{mmHg}$ (SD 10.17).

Conclusion: The pharmacist-led TCC was successfully implemented. Outpatient pharmacists collaborating with ED physicians increased access to HTN management with a positive impact on BP outcomes in an under-resourced population.
\end{abstract}

Keywords: Hypertension, Pharmacist, Collaborative Practice, Emergency Department Physician, Under-resourced

\section{Background}

Although rates of blood pressure (BP) control are increasing, an evaluation from the National Health and Nutrition Examination Survey (NHANES) found that BP is still uncontrolled in $50 \%$ of the US population with hypertension (HTN). ${ }^{1}$ The significant prevalence of HTN (diagnosed and undiagnosed) in African American (AA) men (42.4\%) and women (44\%) 20 years of age or older in the United States is among the highest in the world and arguably the greatest risk factor for cardiovascular health of the AA population. ${ }^{2}$ HTN in AA is more severe and more frequently associated with premature morbidity and mortality from long-term complications including coronary heart disease, heart failure, stroke, and end-stage renal disease. ${ }^{3}$ Targeting

Corresponding Author: Brittany Stewart, RD, PharmD

259 Mack Avenue, Suite 2190

Detroit, MI 48201

Phone: 313-577-9754, Fax: 313-577-5369

Email: brittanystewart@wayne.edu the younger AA population with uncontrolled HTN may be the best strategy to decrease significant long-term complications and cardiovascular risk in this population. Furthermore, the burden of this disease impacts urban AAs with limited access to healthcare disproportionately and can significantly decrease life expectancies. ${ }^{4}$

Several studies have shown that pharmacist-led HTN management improves patient outcomes. ${ }^{5-10}$ Our study explores an innovative pharmacist-led transitional care clinic (TCC) that focuses on HTN management for under-resourced patients in an urban setting. A similar study describes a clinical pharmacist HTN management program in an under-resourced population that takes place in a primary care setting. ${ }^{11}$ However, our study is unique such that it describes a pharmacist-run clinic solely providing HTN management through a CPA without the delegating physician onsite.

Patients with HTN are frequently encountered in the ED, as perhaps the only point of healthcare interaction for some underserved communities. ${ }^{12}$ ED physicians in urban, under- 
resourced medical settings encounter a significant number of patients in the community who are utilizing the ED for primary care needs, such as chronic HTN management. There is a public health need to develop innovative practice models that provide an adjunct to primary care for patients in need of chronic disease state management in these settings. Outpatient and community-based pharmacists are well positioned to fill this need. Pharmacist-led clinics have not been evaluated specifically for the management of hypertensive patients discharged and referred from the ED. Our aim was to successfully implement the outpatient pharmacy TCC in collaboration with ED physicians to provide care for an underresourced urban health population and to evaluate BP control with a pharmacist-led HTN management model.

The objectives of this pilot study were to implement the TCC and to evaluate outpatient pharmacist management of essential HTN for under-resourced patients.

\section{Setting}

The Detroit Medical Center (DMC) Sinai Grace Hospital (SGH) is a community academic hospital located in Northwest Detroit. The TCC was located adjacent to the outpatient pharmacy on the first floor of SGH. One clinical pharmacist, who is also shared faculty, staffed the clinic along with student pharmacists and pharmacy residents. The pharmacist's role at the TCC was to provide medication therapy management and outpatient pharmacy transitions of care services. The pharmacist provided comprehensive HTN management under a collaborative practice agreement (CPA) with ED physicians. The CPA allowed for the pharmacist to initiate, modify, or discontinue antihypertensive therapy and to use clinical judgment to make individualized therapeutic decisions. The CPA was written collaboratively and approved by the DMC legal department. Our TCC model provides expanded pharmacist services that increase access to care and HTN management for underresourced patients with uncontrolled HTN.

\section{Methods}

This was a single arm, prospective, pilot study to recruit eligible patients from the DMC SGH ED and Detroit community health events from May 2017 to August 2018. Patients were screened and consented by the ED research team for the following inclusion criteria: (1) 18 to 60 years old, (2) blood pressure reading $>140 / 90 \mathrm{~mm} / \mathrm{HG}$, (3) previous diagnosis of HTN, (4) lack of consistent primary care relationship (less than 1 visit in 6 months), and (5) ability to provide informed consent. The goal was to recruit patients with essential HTN and low chronicity of comorbid diseases. Patients were excluded for (1) diagnosis of greater than 2 chronic conditions in addition to HTN, (2) chronic kidney disease, congestive heart failure, uncontrolled diabetes, or any condition that limits activities of daily life, (3) terminal illness, (4) resident in an assisted living or skilled nursing facility, and (5) pregnancy. Eligible patients were referred to follow up for HTN management at the TCC with the pharmacist and received a \$20 gift card at each visit for incentive. The 6-month intervention included a series of five visits: visit one, at two weeks (visit two), at one month (visit three), at three months (visit four), and at six months (visit five). At each visit, the pharmacist obtained an average BP value from three measurements taken with a BpTru automated monitor and also discussed medication adherence and lifestyle interventions (nutrition, exercise, smoking cessation). Additionally, a pointof-care lipid panel was completed at visits one and five. As shown in Figure 1, the pharmacist initiated and modified antihypertensive therapy in accordance with the CPA following the Joint National Committee Guidelines for Management of Hypertension $8 .{ }^{13}$ Each patient received individualized care with an emphasis on overcoming medication access issues and other medication-related barriers (cost of medications, prescription delivery, etc.) to HTN management. TCC clinic visits were documented in the DMC electronic health record. At each visit, the pharmacist encouraged patients to reengage in a primary care relationship and the importance for chronic HTN management. At the conclusion of the study intervention, patients were given information for transitioning to follow up with a PCP for long-term HTN management.

Study data were collected and managed using REDCap electronic data capture tools hosted at Wayne State University. ${ }^{14}$ The primary outcome measure was the change in SBP and DBP from visit to visit throughout the study intervention. While ED BP values are reported, baseline BP values for the purpose of the study intervention began at TCC visit one with the pharmacist. Difference in BP values was evaluated using the Wilcoxon Signed Ranks test. Descriptive statistics were used to describe demographic data. There was loss to follow up over the course of the five-visit intervention and statistical imputation methods were used, but did not affect statistical analysis. We analyzed the data in three cohorts: (1) patients who completed at least one TCC pharmacy visit; (2) patients who completed at least three TCC pharmacy visits; and (3) patients who completed all five TCC pharmacy visits. For patients that did not complete all five visits, the BP data was imputed up through visit five. Data were analyzed using SPSS version 26. The Wayne State University and DMC Institutional Review Board approved the protocol prior to study initiation.

\section{Results}

One hundred and sixteen patients were enrolled in the study; $44(37.9 \%)$ patients presented to TCC visit one, $30(25.9 \%)$ completed at least three visits, and $16(13.8 \%)$ patients completed all five TCC study visits with the pharmacist. (Figure 2.) As anticipated, our study cohort was predominantly $A A$ (97.8\%, SD 43) which is representative of the patient population, $47.8 \%$ (SD 21) male, 64\% (SD 28) current smoker, and about half $(52.3 \%$, SD 23$)$ of patients were not taking any antihypertensive medications at enrollment. All patient baseline characteristics are presented in Table 1. 
Blood pressure values were obtained in the ED, however, there are no BP values to report prior to TCC visit one for patients recruited at community screening events. Table 2 displays the $B P$ values for the three cohorts across all study visits. For cohorts 2 and 3, there was significant reduction in SBP and DBP between TCC visits one and two and the reduction was maintained through five visits for patients that remained in the study. The greatest reduction in SBP between visits one [SBP $162 \mathrm{mmHg}$ (SD 27.49)] and visit two [SBP $140 \mathrm{mmHg}$ (SD 17.49)] occurred in cohort 3 , resulting in a change in SBP of $-22 \mathrm{mmHg}$ $(p=0.015)$. Patients who completed the first three visits $(n=30)$ were close to BP goal ( $<140 / 90 \mathrm{mmHg}$ ) with an average SBP 141 $\mathrm{mmHg}$ (SD 16.60) and DBP $92 \mathrm{mmHg}$ (SD 10.91). Patients who completed all five visits $(n=16)$ showed a significant change in SBP of $-23 \mathrm{mmHg}(\mathrm{p}=0.002)$ from visit one to visit five and 100 $\%$ achieved BP goal with an average SBP $139 \mathrm{mmHg}$ (SD 19.33) and DBP $90 \mathrm{mmHg}$ (SD 10.17) (Figure 3). There was a trend in SBP reduction across all of the completed visits of all the cohorts with most significant and sustained reduction in cohort 3 (Figure 4).

There was continued loss to follow up as the study visits progressed with $34 \%$ of patients that came for visit one dropped out by visit three. Additionally, the dropout rate between visit three to four was $23 \%$ and from visit four to five was $30 \%$ with a total dropout rate of $64 \%$ from visit one to visit five.

\section{Discussion}

This study demonstrated that we found it feasible to implement a pharmacist-led TCC clinic for patients in an under-resourced population that are discharged from the ED or identified at community screening events and lacking in primary care. Moreover, we showed the ability to achieve a significant reduction in SBP and DBP by visit three and reach BP goal $(<140 / 90 \mathrm{mmHg}$ ) by visit five for those who completed all visits. The results of our study confirm the positive impact that pharmacists can have on increasing access to care and HTN management.

Our study cohort was largely AA which is important as AA patients in medically underserved communities face numerous barriers to accessing health care making them vulnerable to long-term complications of uncontrolled HTN. For many such patients, the ED has become a de facto primary care resource. ${ }^{15}$ However, effective HTN control requires on-going, long-term management and ED's are not well equipped to deliver this nor are they designed to effectively serve as transition points for referral to primary care. ${ }^{16}$ Patients with low socioeconomic status, under insured, or lacking in a primary care provider are at greatest risk for loss to follow up post-ED visits. ${ }^{17}$ The patients in our study are reflective of a high-risk population for loss to follow up with lacking a primary care relationship. However, almost $40 \%$ of enrolled patients came to the TCC for at least one visit with the pharmacist. The pharmacist made several attempts to reach the dropout patients by phone and was unsuccessful, many of the phone numbers were no longer in service or had been changed. There were three study visits within the first month and then a 2-month gap from visit three to four and four to five which may have contributed to the dropout. The significant decrease in BP from visit one to three and the pharmacist providing antihypertensive prescriptions to the patient may have influenced lack of follow up beyond visit three. The loss to follow up in our study population may have also been influenced by factors including time, transportation, and knowledge barriers.

Community-based care models, including our TCC model, in under-resourced communities often face difficulty with reproducibility and widespread implementation due to cost, lack of medical insurance, transportation barriers, and general health care access barriers. ${ }^{18}$ We faced barriers including: difficulty contacting and scheduling patients, transportation issues, missed appointments, and dropout as previously discussed. We were able to provide patients with taxi service if they had no transportation to the appointment. Institutions in under-resourced communities face challenges to develop adjunct primary care models to alleviate the burden of HTN and decrease disparities in health outcomes. In recent years, health outreach and community-based programs aimed at combatting HTN in the AA population in locations such as barbershops, churches, and beauty salons resulted in positive results. ${ }^{18}$ Notably, the largest of these "barbershop studies" utilized clinical pharmacists working under a CPA with primary care providers and resulted in significant and sustained BP reduction. ${ }^{19,} 20$

Pharmacist-prescriber collaboration will be essential to expand pharmacist-led services and continue to increase access to healthcare, especially in under-resourced populations. The CDC encourages advancing team-based care through CPAs to improve outcomes for chronic disease state management such as HTN. ${ }^{21}$ While there are several published pharmacistphysician collaborative models for HTN management in a variety of pharmacy settings, ${ }^{22}$ to our knowledge, this is the first study to evaluate a model with an outpatient pharmacist in collaboration with ED physicians. Although we did not measure the impact on ED visits or primary care establishment following the study intervention, this would be beneficial to evaluate in the future. This innovative approach could represent a viable mechanism to address the long-standing challenges with transitioning ED patients with uncontrolled HTN to effective pharmacist-led outpatient care.

\section{Limitations}

The results of our study should be considered within the context of various limitations. This study excluded patients with greater than 2 chronic medical conditions in addition to HTN and specific conditions that increase cardiovascular risk due to the complex nature of medical management of the patients and the focus of our study was to target a younger population with uncontrolled essential HTN. The study sample 
size was small, although this does not lessen the significance found in SBP and DBP reduction throughout the intervention. The study intervention period was limited and we do not know the long-term outcomes the intervention had on patients. This was a one-arm pilot study with no control group or historical data for comparison. Patients received incentive for each visit and this may have influenced the number of patients that attended follow up appointments. A larger scale, longer duration, randomized controlled trial with a comparison of our intervention to usual care would further validate the results of our pilot study.

\section{Conclusion}

Outpatient pharmacists can successfully collaborate with ED physicians and have a positive impact on BP outcomes in an under-resourced population. Our innovative pharmacy practice model represents a novel collaboration between ED physicians and pharmacists that can streamline the ability of providers to help reduce the impact of uncontrolled HTN, while addressing an unmet need for patients who rely on the ED for primary care.

\section{Author Contributions}

Brittany Stewart: Conceptualization, Methodology, Validation, Investigation, Resources, Data Curation, Writing - Original Draft, Writing - Review and Editing, Visualization, Supervision, Project Administration, Funding Acquisition Aaron Brody: Conceptualization, Methodology, Validation, Visualization, Supervision Candice Garwood: Writing - Original Draft, Writing - Review and Editing, Visualization Liying Zhang: Formal analysis Phillip Levy: Conceptualization, Methodology, Validation, Writing - Review and Editing, Visualization, Supervision, Project Administration

Funding Support: The study was funded by an internal faculty research grant awarded to Dr. Stewart from the Eugene Applebaum College of Pharmacy and Health Sciences at Wayne State University and the National Association of Chain Drug Stores (NACDS). NACDS had no involvement with the research conducted or in the writing of the publication.

Disclosures of Conflict of Interest: None of the authors have a declaration of conflict of interest to report.

\section{References}

1. Gums TH, Uribe L, Vander Weg MW, James P, Coffey C, Carter BL. Pharmacist intervention for blood pressure control: medication intensification and adherence. Journal of the American Society of Hypertension : JASH 2015;9:569-578.

2. Carnethon MR, Pu J, Howard G, et al. Cardiovascular Health in African Americans: A Scientific Statement From the American Heart Association. Circulation 2017;136:e393-e423.

3. Still $\mathrm{CH}$, Craven TE, Freedman BI, et al. Baseline characteristics of African Americans in the Systolic Blood Pressure Intervention Trial. Journal of the American Society of Hypertension : JASH 2015;9:670679.

4. Flack JM, Ferdinand KC, Nasser SA. Epidemiology of hypertension and cardiovascular disease in African Americans. Journal of clinical hypertension (Greenwich, Conn) 2003;5:5-11.

5. Glynn LG, Murphy AW, Smith SM, Schroeder K, Fahey T. Interventions used to improve control of blood pressure in patients with hypertension. The Cochrane database of systematic reviews 2010:Cd005182.

6. Houle SK, Chuck AW, McAlister FA, Tsuyuki RT. Effect of a pharmacist-managed hypertension program on health system costs: an evaluation of the Study of Cardiovascular Risk Intervention by PharmacistsHypertension (SCRIP-HTN). Pharmacotherapy 2012;32:527-537.

7. Tsuyuki RT, Houle SK, Charrois TL, et al. Randomized Trial of the Effect of Pharmacist Prescribing on Improving Blood Pressure in the Community: The Alberta Clinical Trial in Optimizing Hypertension (RxACTION). Circulation 2015;132:93-100.

8. Wal P, Wal A, Bhandari A, Pandey U, Rai AK. Pharmacist involvement in the patient care improves outcome in hypertension patients. Journal of research in pharmacy practice 2013;2:123-129.

9. Weber CA, Ernst ME, Sezate GS, Zheng S, Carter BL. Pharmacist-physician comanagement of hypertension and reduction in 24-hour ambulatory blood pressures. Archives of internal medicine 2010;170:1634-1639.

10. Zillich AJ, Sutherland JM, Kumbera PA, Carter BL. Hypertension outcomes through blood pressure monitoring and evaluation by pharmacists (HOME study). Journal of general internal medicine 2005;20:1091-1096.

11. Wooley AC, Brooks AD, Stacy ZA. Effect of a Clinical Pharmacist-Managed Service on Blood Pressure in an Underserved Population With Resistant Hypertension. J Pharm Technol 2016;32:135-142. 
12. Brody A, Rahman T, Reed B, et al. Safety and efficacy of antihypertensive prescription at emergency department discharge. Academic emergency medicine : official journal of the Society for Academic Emergency Medicine 2015;22:632-635.

13. James PA, Oparil S, Carter BL, et al. 2014 evidencebased guideline for the management of high blood pressure in adults: report from the panel members appointed to the Eighth Joint National Committee (JNC 8). JAMA 2014;311:507-520.

14. RedCap.

15. Cheung PT, Wiler JL, Lowe RA, Ginde AA. National study of barriers to timely primary care and emergency department utilization among Medicaid beneficiaries. Annals of emergency medicine 2012;60:4-10.e12.

16. Baumann BM, Cline DM, Cienki JJ, Egging D, Lehrmann JF, Tanabe P. Provider self-report and practice: reassessment and referral of emergency department patients with elevated blood pressure. American journal of hypertension 2009;22:604-610.

17. Naderi S, Barnett B, Hoffman RS, et al. Factors associated with failure to follow-up at a medical clinic after an ED visit. Am J Emerg Med 2012;30:347-351.

18. Maraboto C, Ferdinand KC. Update on hypertension in African-Americans. Progress in cardiovascular diseases 2020;63:33-39.

19. Victor RG, Blyler CA, Li N, et al. Sustainability of Blood Pressure Reduction in Black Barbershops. Circulation 2019;139:10-19.

20. Victor RG, Lynch K, Li N, et al. A Cluster-Randomized Trial of Blood-Pressure Reduction in Black Barbershops. N Engl J Med 2018;378:1291-1301.

21. Control CfD. Advancing Team-Based Care Through Collaborative Practice AgreementsA Resource and Implementation Guide for Adding Pharmacists to the Care Team. Atlanta, GA: Centers for Disease Control and Prevention, U.S. Department of Health and Human Services; 2017. 2017.

22. Stewart B, Brody A, Krishnan AC, Brown SK, Levy PD. An Unmet Need Meets an Untapped Resource: Pharmacist-Led Pathways for Hypertension Management for Emergency Department Patients. Current hypertension reports 2019;21:61. 